\title{
Enzymatic Synthesis of the C-Glycosidic Moiety of Nogalamycin R
}

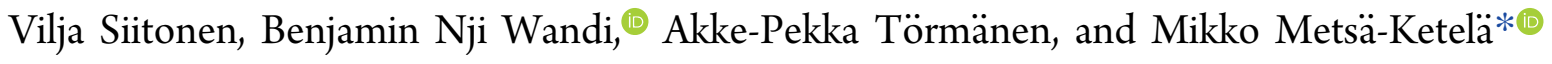 \\ Department of Biochemistry, University of Turku, FIN-20014 Turku, Finland
}

\section{Supporting Information}

ABSTRACT: Carbohydrate moieties are essential for the biological activity of anthracycline anticancer agents such as nogalamycin, which contains L-nogalose and L-nogalamine units. The former of these is attached through a canonical $O$-glycosidic linkage, but the latter is connected via an unusual dual linkage composed of $\mathrm{C}-\mathrm{C}$ and $\mathrm{O}$-glycosidic bonds. In this work, we have utilized enzyme immobilization techniques and synthesized Lrhodosamine-thymidine diphosphate (TDP) from $\alpha$-D-glucose-1TDP using seven enzymes. In a second step, we assembled the dual linkage system by attaching the aminosugar to an anthracycline aglycone acceptor using the glycosyl transferase

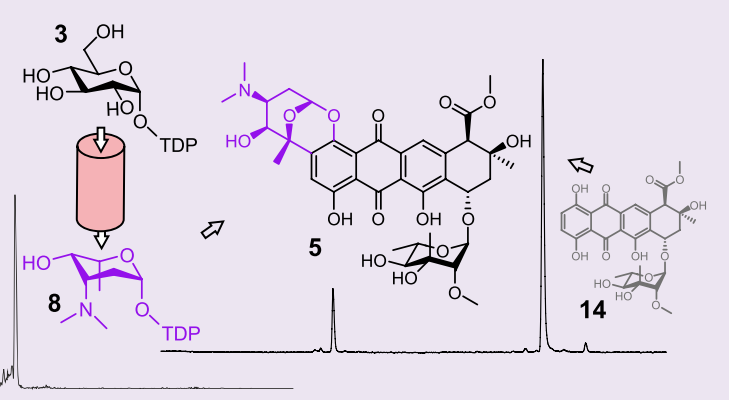
SnogD and the $\alpha$-ketoglutarate dependent oxygenase SnoK. Furthermore, our work indicates that the auxiliary P450-type protein SnogN facilitating glycosylation is surprisingly associated with attachment of the neutral sugar L-nogalose rather than the aminosugar L-nogalamine in nogalamycin biosynthesis.

M any natural products owe their biological activity to carbohydrate units attached to aglycones. ${ }^{1}$ Examples of microbial secondary metabolites of medical relevance include the anticancer agent doxorubicin, ${ }^{2}$ the antibiotic erythromycin, ${ }^{3}$ and the anthelmintic drug avermectin. ${ }^{4}$ The most common mode of attachment is via an $\mathrm{O}$-glycosidic linkage, which is unfortunately susceptible to hydrolysis, ${ }^{5}$ as are carbohydrate units appended through $\mathrm{N}$ - or $\mathrm{S}$-glycosylation. Chemically more robust $C$-glycosylation has been reported in selected instances. ${ }^{6}$ Of particular interest is nogalamycin $(\mathbf{1}$, Figure 1$),{ }^{7}$ produced by Streptomyces nogalater ATCC 27451, where the aminosugar nogalamine is attached both via an $\mathrm{O}$-glycosidic linkage and an additional carbon-carbon bond.

The great diversity of naturally occurring carbohydrates has raised considerable interest in their biosynthesis, and several pathways have been elucidated in vitro with purified enzymes. $^{8-13}$ The most common starting material is $\alpha$-Dglucose-1-phosphate (2, Scheme 1), which is activated further by nucleotidyl transferases to generate TDP-glucose (3, Scheme $1)$. Most glycosylated bacterial secondary metabolites are derived from TDP-6-deoxyhexoses, and the second biosynthetic step catalyzed by TDP-D-glucose-4,6-dehydratase is typically also conserved. After formation of TDP-4-keto-6-deoxy- $\alpha$-Dglucose (4, Scheme 1), the pathways diverge to various C2, C3, or C4 deoxysugars and aminosugars. ${ }^{13,14}$

The C-glycosidic unit of $\mathbf{1}$ has raised considerable interest, and the fragment was chemically synthesized soon after the stereochemistry of the natural product was confirmed in $1983 .{ }^{15}$ However, understanding the biosynthesis and attachment of nogalamine has been more challenging. Characterization of the gene cluster and heterologous expression studies in S. albus led to the isolation of nogalamycin $\mathrm{R}$ ( 5 , Figure 1 ), where $\mathrm{L}$ rhodosamine ( $4^{\prime \prime}$-epi-2"-deoxy-nogalamine) is attached via the

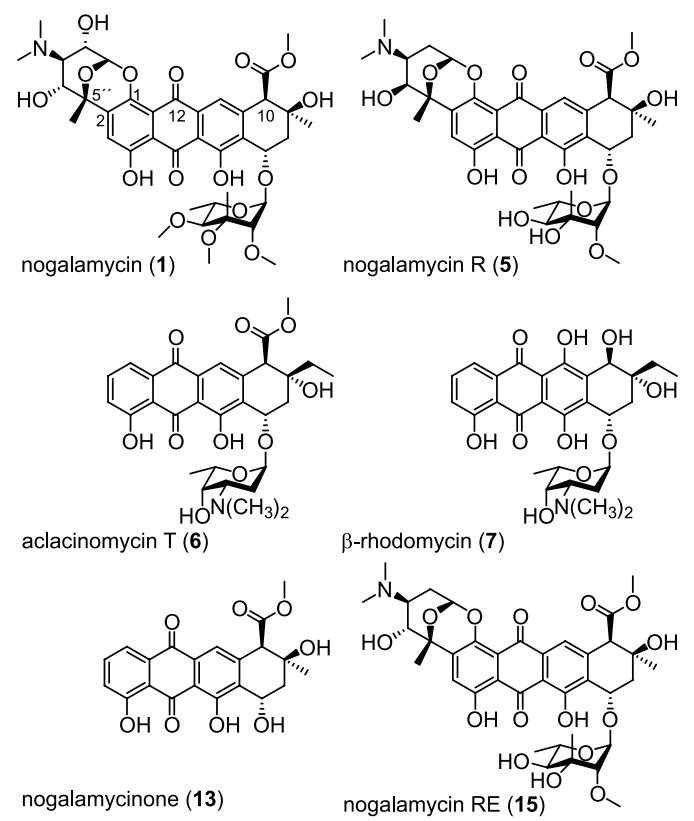

Figure 1. Chemical structures of the anthracyclines nogalamycin (1), nogalamycin $\mathrm{R}$ (5), aclacinomycin $\mathrm{T}$ (6), $\beta$-rhodomycin (7), nogalamycinone (13), and nogalamycin RE (15) relevant to the study.

dual linkage system to the anthracycline aglycone. ${ }^{16}$ Recent studies have verified that the $\mathrm{C} 2-\mathrm{C} 5$ " bond and $\mathrm{C} 4$ " epimerization are catalyzed by two related $\alpha$-ketoglutarate and

Received: July 12, 2018

Accepted: August 16, 2018

Published: August 16, 2018 
Scheme 1. Model for the Biosynthesis of TDP-L-Rhodosamine and Its Dual Attachment to the Anthracycline Scaffold ${ }^{a}$

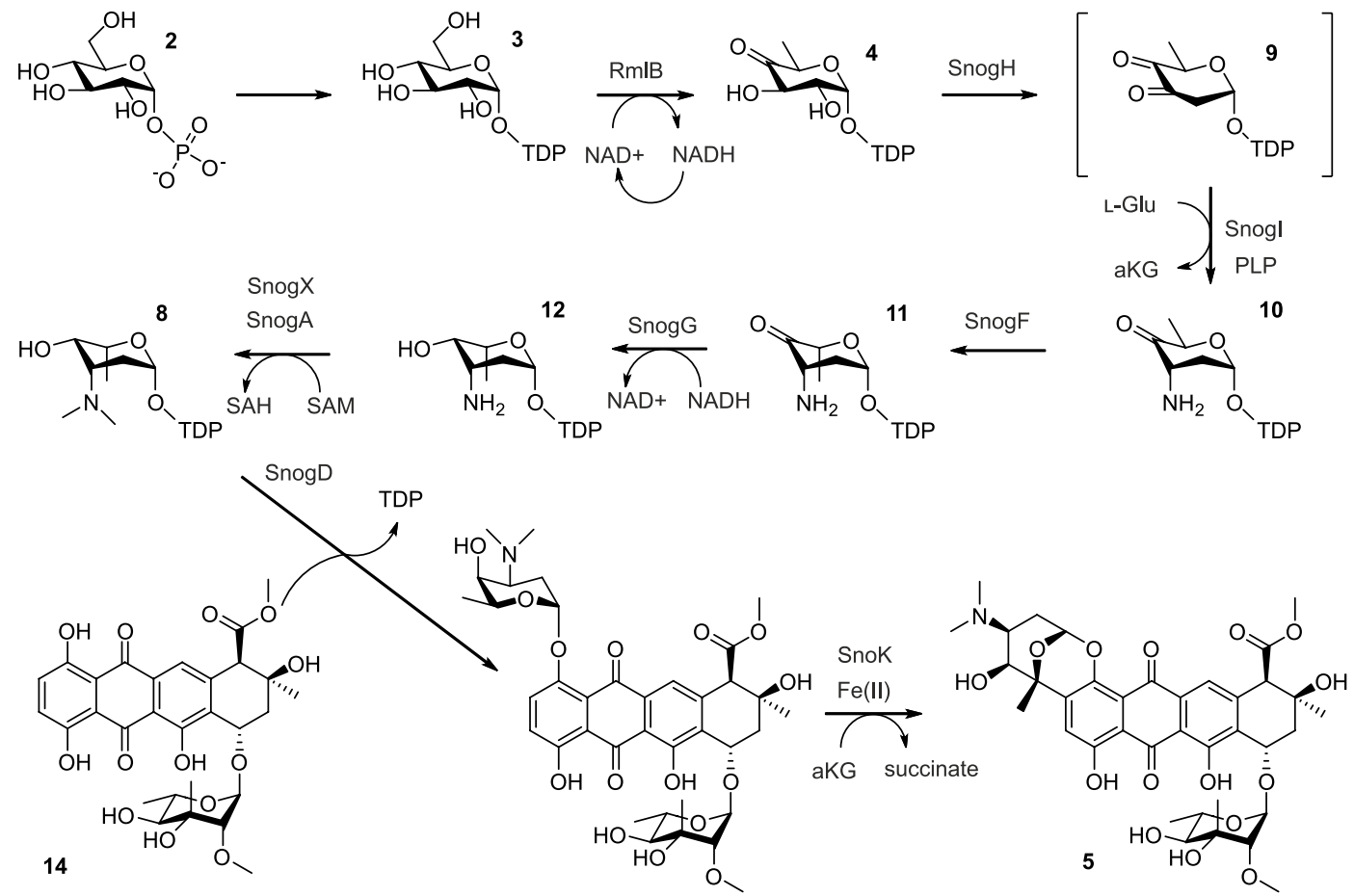

${ }^{a_{\mathrm{TDP}}}$, thymidine diphosphate; NAD+, nicotinamide adenine dinucleotide, oxidized; NADH, nicotinamide adenine dinucleotide, reduced; PLP, pyridoxal 5'-phosphate; L-Glu, L-glutamate; SAM, S-adenosyl-L-methionine; SAH, S-adenosyl-L-homocysteine; aKG, $\alpha$-ketoglutarate.

nonheme iron-dependent enzymes SnoK and SnoN, respectively. ${ }^{17}$ In this Letter, we report further molecular genetic characterization of the nogalamycin gene cluster and confirm TDP-L-rhodosamine as a true pathway intermediate by enzymatic synthesis.

L-Rhodosamine is a commonly occurring carbohydrate in anthracyclines such as aclacinomycins (6, Figure 1), rhodomycins (7, Figure 1), cosmomycins, and cytorhodins. ${ }^{18-20}$ To the best of our knowledge, the enzymatic synthesis of TDP-Lrhodosamine (8, Scheme 1$)$ has not been reported, although it has been chemically synthesized from TDP-L-daunosamine via $\mathrm{N}$-dimethylation. ${ }^{21}$ The bioinformatic analysis of the nogalamycin gene cluster was complicated by the existence of additional gene products, which have not been experimentally verified, that are involved in the biosynthesis of L-nogalose, the neutral carbohydrate at $\mathrm{C} 7$ in $\mathbf{1}$. The nucleotidyl transferase SnogJ and the 4,6-dehydratase SnogK may be shared in the biosynthesis of the two carbohydrates for generation of the putative last common intermediate 4 . We surmised that the next step would be catalyzed by the 2,3-dehydratase SnogH leading to the formation of TDP-3,4-diketo-2,6-dideoxy- $\alpha$-D-glucose (9, Scheme 1), which would allow transamination by the pyridoxal 5 '-phosphate (PLP)-dependent SnogI to generate TDP-3amino-4-keto-2,3,6-trideoxy- $\alpha$-D-glucose (10, Scheme 1). The pathway could then proceed through 5-epimerization by SnogF (11, Scheme 1) and 4-ketoreduction. Previous models have suggested that the 4-ketoreduction leads to TDP-L-acosamine, ${ }^{22}$ which has the same stereochemistry at $\mathrm{C} 4$ as the end product Lnogalamine, but we hypothesized that SnogG would catalyze the formation of TDP-L-daunosamine (12, Scheme 1) instead, in a manner similar to daunorubicin and aclacinomycin biosynthesis. ${ }^{18}$ Finally, the cluster harbors two homologous genes that code for methyl transferases, SnogX and SnogA (54\% sequence identity), that could be responsible for the generation of TDP-Lrhodosamine (8, Scheme 1$)$.

We opted to utilize 3 as the starting material for the synthesis and cloned overexpression constructs for heterologous production of the required proteins in Escherichia coli TOP10. Most of the proteins were cloned from the nogalamycin pathway, but $\operatorname{snogK}$ was replaced with the orthologous $r m l B$ originating from E. coli K12. ${ }^{23}$ The proteins were produced as $\mathrm{N}$ terminally histidine tagged enzymes, which allowed single-step purification to near homogeneity by affinity chromatography (Figure S1).

We proceeded to perform one-pot enzymatic synthesis for production 8 with the seven enzymes. One of the challenges in multienzyme catalysis is to find conditions where all components are functional. In our case, the solubility of SnogG and SnogA was poor, which led to precipitation of the enzymes over time and, for instance, prevented the use of centrifugal concentrators to reach micromolar protein concentrations. To solve this issue, we immobilized the seven enzymes to TALON affinity beads and conducted the reactions under gentle shaking at $23^{\circ} \mathrm{C}$ in a suitable reaction buffer, with $10 \mathrm{mM}$ of 3, $10 \mu \mathrm{M}$ of $\mathrm{SnogH}, 30 \mu \mathrm{M}$ of the other proteins, and $90 \mu \mathrm{M}$ to $6 \mathrm{mM}$ of the various cofactors and cosubstrates. The benefits of the approach were many-fold, since in addition to improving stability issues, protein immobilization techniques have been shown to enhance overall catalysis by increasing local enzyme concentrations. $^{24}$

Monitoring of the reactions by LC-MS revealed formation of the expected ion $8\left([\mathrm{M}-\mathrm{H}]^{-}\right.$; calcd, 558.1; found, 558.2) as the product. All of the substrate 3 was consumed, but minor quantities of various putative intermediates could be observed (Figure S2). Surprisingly, the presence of both methyl transferases was not essential for the reaction and 8 could be detected in reactions with either SnogX (Figure 2A) or SnogA 

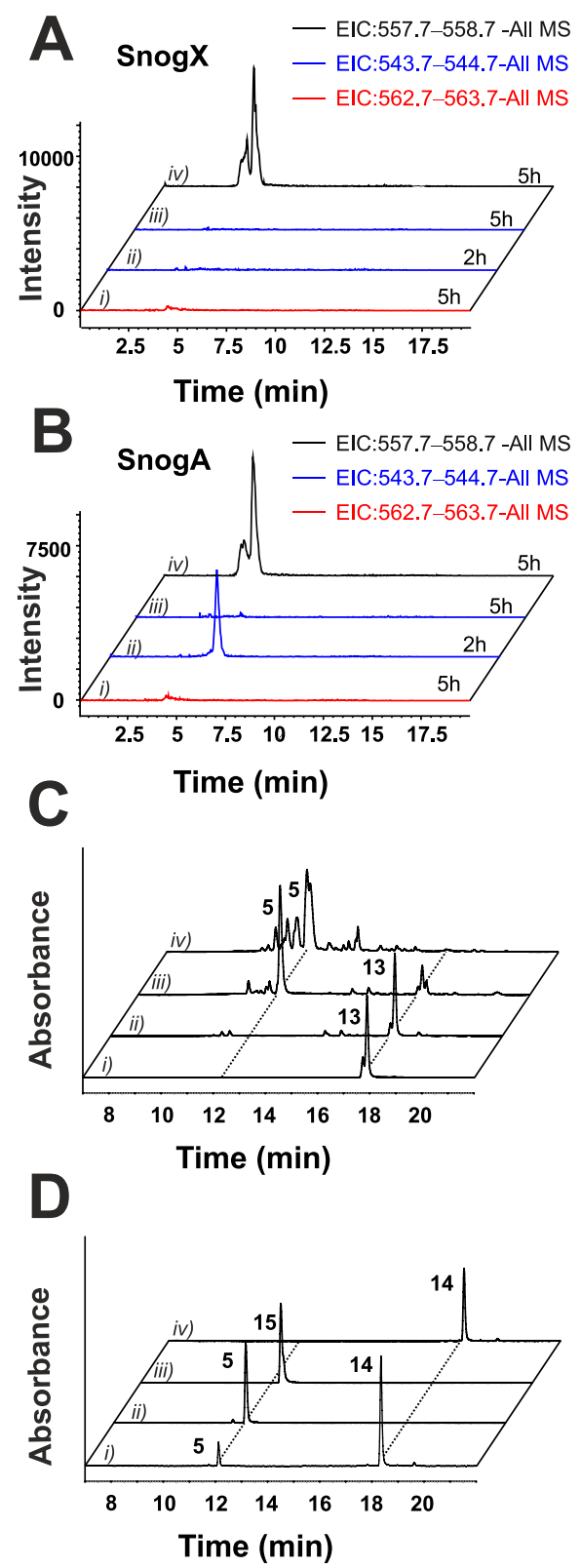

Figure 2. LC-MS and HPLC analysis of in vitro and in vivo reaction products. (A) LC-MS analysis of the in vitro sugar enzymatic formation of TDP-L-rhodosamine using $\operatorname{SnogX}$ as the methyltransferase. (i) Extracted ion chromatogram (EIC) for the used substrate 3 after a $5 \mathrm{~h}$ reaction. (ii) EIC for the monomethylated product in a $2 \mathrm{~h}$ reaction. (iii) EIC for the monomethylated product in a $5 \mathrm{~h}$ reaction. (iv) EIC for the dimethylated product 8 in a $5 \mathrm{~h}$ reaction. (B) LC-MS analysis of the in vitro sugar enzymatic formation of TDP-L-rhodosamine using SnogA as the methyltransferase. (i) EIC for the used substrate 3 after a $5 \mathrm{~h}$ reaction. (ii) EIC for the monomethylated product in a $2 \mathrm{~h}$ reaction. (iii) EIC for the monomethylated product in a $5 \mathrm{~h}$ reaction. (iv) EIC for the dimethylated product 8 in a 5 h reaction. (C) HPLC analysis of the in vivo products shown at $430 \mathrm{~nm}$. (i) Standard for 13. (ii) Extract from the strain S. albus/pSno $\Delta \mathrm{gN}$, (iii) S. albus $/ \mathrm{pSno} \Delta \mathrm{gN}+\mathrm{pIJTsyngN}$, and (iv) S.albus/pSnogaori and (D) HPLC analysis of the in vitro products shown at $430 \mathrm{~nm}$. (i) enzymatic synthesis of nogalamycin R (5). (ii) Standard for 5. (iii) Standard for 15. (iv) Used substrate 14.

(Figure 2B) alone. After $2 \mathrm{~h}$ of incubation, the reaction with SnogX produced solely $\mathbf{8}$, whereas a monomethylated intermediate could also be observed with SnogA. However, this intermediate was further converted to 8 after extending the reaction time to $5 \mathrm{~h}$ (Figure $2 \mathrm{~B}$ ). The relative activity measurements imply that SnogA is poor in converting monomethylated intermediates to 8 . This indicates that the biological role of SnogA in nogalamycin biosynthesis may be to carry out the first methylation reaction, followed by the second methylation step by $\operatorname{SnogX}$, even though both enzymes are capable of catalyzing dimethylations.

The glycosyl transferase SnogD has been identified to be responsible for the glycosylation at $\mathrm{C} 1,{ }^{16}$ and the crystal structure of SnogD has been determined, ${ }^{25}$ but to date the reaction has only been probed in vitro in the reverse direction. Many glycosyl transferases have been shown to require the aid of P450-like enzymes for catalysis, ${ }^{26}$ such as AknS/AknT in aclacinomycin biosynthesis, where they are responsible for the transfer of L-rhodosamine to C7 of the aglycone. ${ }^{27}$ Although the exact function of these auxiliary proteins is unknown, structural studies have indicated their involvement in allosteric activation of glycosyl transferases. ${ }^{26}$ Generally, these proteins have been implicated to be involved specifically in the transfer of aminosugars. $^{28-31}$ To investigate whether the corresponding gene $\operatorname{snog} N$ is involved in the transfer of $\mathbf{8}$, we inactivated the gene from the cosmid $\mathrm{pSnogaori}$ by $\mathrm{RED} / \mathrm{ET}$ recombineering in E. coli and introduced the engineered cosmid pSno $\Delta \mathrm{gN}$ to $S$. albus by conjugation. Analysis of culture extract revealed surprisingly that the main metabolite produced was nogalamycinone (13, Figure 2B), indicating that SnogN may be working in conjunction with the TDP-L-nogalose transferase SnogE. This would imply that the requirement for auxiliary proteins has more to do with the chemical structure of the aglycone acceptor molecule rather than the donor TDP-aminosugar in nogalamycin biosynthesis. To rule out polar effects, the mutation to snogN was complemented with an intact copy of the gene, which restored production of the double glycosylated metabolite $\mathbf{5}$ (Figure 2C).

In order to unequivocally confirm the identity of the synthesized nucleotide sugar as $\mathbf{8}$, we continued the enzymatic synthesis and attached the TDP-carbohydrate to a monoglycosylated nogalamycin scaffold (14, Scheme 1), since double glycosylated nogalamycin standards were available from previous studies. ${ }^{17}$ The expected product 5 was observed after incubation of the two substrates in the presence of $7 \mu \mathrm{M}$ of the glycosyl transferase $\operatorname{SnogD}^{16}$ and $2.5 \mu \mathrm{M}$ of the carbocyclase SnoK, ${ }^{17} \alpha$-ketoglutarate, $\mathrm{Fe}(\mathrm{II})$ and ascorbate (Figure 2D). The experiment verified the stereochemistry of the attached aminosugar, since the 4 "-epimer (15, Figure 1) of 5 has a distinct retention time under our analytical conditions (Figure 2D). ${ }^{17}$ To the best of our knowledge, this represent the first example of enzymatic synthesis of a TDP-aminosugar and its attachment to a polyketide scaffold.

In summary, this work describes a two-pot reaction of nine enzymes associated with TDP-L-rhodosamine (8) formation and attachment in biosynthesis of the anthracycline nogalamycin. It provides an instructive example of how microbial secondary metabolism pathways have evolved in a circuitous manner; both (i) the initial 4-ketoreduction by SnogG and late stage inversion of stereochemistry at $\mathrm{C}^{\prime \prime}$ by SnoN and (ii) the loss of the $\mathrm{C}^{\prime \prime}$ hydroxyl group by $\mathrm{SnogH}$ and subsequent rehydroxylation putatively by SnoT are unnecessary from the perspective of a synthetic chemist.

\section{METHODS}

Strain and Culture Conditions. Escherichia coli TOP 10 (Invitrogen) was used as the cloning host. E. coli $\mathrm{K} 12$ was used as a 
host for engineering the cosmid pSnogaori and ET12567/pUZ8002 $2^{32}$ for intergeneric conjugation to Streptomyces albus. ${ }^{33}$ Streptomyces cultivations were performed in NoS-soyE $1,{ }^{16}$ tryptone soya broth (TSB; Oxoid), R2 yeast extract (R2YE), and mannitol soya flour medium (MS) ${ }^{32}$ E. coli strains were cultivated in Luria-Bertoli or $2 \times$ yeast extract/tryptone medium $(2 \mathrm{xTY})$. Used antibiotics were ampicillin $\left(50 \mu \mathrm{g} \mathrm{mL}{ }^{-1}\right.$, Sigma-Aldrich), nalidixic acid $\left(500 \mu \mathrm{g} \mathrm{mL}^{-1}\right.$, Sigma-Aldrich), kanamycin (25 $\mu \mathrm{g} \mathrm{mL}^{-1}$, Sigma-Aldrich), chloramphenicol $\left(25 \mu \mathrm{g} \mathrm{mL}^{-1}\right.$, Sigma-Aldrich $)$, and apramycin $(50 \mu \mathrm{g}-1250 \mu \mathrm{g}$ $\mathrm{mL}^{-1}$, Sigma-Aldrich).

Cloning and General DNA Techniques. Standard Streptomyces techniques were used. ${ }^{32}$ Commercial kits were used for recovering DNA from agarose gels and for plasmid isolation (Thermo Scientific and E.Z.N.A, Omega Bio-Tek, respectively). DNA modifying enzymes were purchased from Thermo Scientific. The genes of interest were amplified by PCR using Phusion (New England Biolabs) and pSnogaori ${ }^{16}$ or boiled E. coli K12 cells as the template. The PCR products were digested and cloned to the modified pBADHisBplasmid $^{34}$ as BglII-EcoRI, BglII-HindIII-, or PstI-HindIII-fragments. The list of primers is given in Table S1. The glycosyl transferase snogD was cloned as a truncated version as described earlier. ${ }^{16}$ The constructs were verified by sequencing (Eurofins MWG Operon).

The sno $\Delta \mathrm{gN}$ gene inactivation mutant was made by deleting $\operatorname{snogN}$ (1227 bp) from the cosmid pSnogaori by using the $\lambda$ Red recombinase system. ${ }^{35}$ PCR was performed using DyNAzyme II (New England Biolabs) and pSnogaori as the template and the primers given in Table S1. First, a chloramphenicol resistance gene was introduced instead of the $\operatorname{snogN}$ gene and subsequently removed by pFLP2. ${ }^{36}$ The construct was conjugated to S.albus from E. coli ET12567/pUZ8002, ${ }^{32}$ resulting in the strain $S$. albus $/ \mathrm{pSno} \Delta \mathrm{gN}$. The strain $S$. albus $/ \mathrm{pSno} \Delta \mathrm{gN}$ was complemented with a synthetically ordered native $\operatorname{snogN}$ with additional native promoter sequence upstream of the gene ( $83 \mathrm{bp}$ ) (Geneart). The synthetic gene was cloned to the vector pIJT486, ${ }^{16}$ amplified in S. lividans TK24 and introduced to $S$. albus/pSno $\Delta \mathrm{gN}$ by protoplast transformation resulting in strain $S$. albus/pSno $\Delta \mathrm{gN}+$ pIJTsyngN. The complemented strain was compared to $S$. albus/ pSnogaori. ${ }^{16}$ The strains were grown for 6 days in NoS-soyE $1,{ }^{16}$ after which absorbent (LXA1180, Sunresin, $20 \mathrm{~g} \mathrm{l}^{-1}$ ) was added to the culture. The compounds produced were extracted after 1 day by using methanol. The extract was analyzed by SCL-10Avp HPLC with an SPD-M10Avp diode array detector (Shimadzu) with a reversed-phase column (Phenomenex Kinetics, $2.6 \mu \mathrm{m}, 4.6 \times 100 \mathrm{~mm}$ ) using a gradient from $15 \%$ acetonitrile with $0.1 \%$ formic acid to $100 \%$ acetonitrile. The standard for 13 was obtained from earlier studies. ${ }^{16}$

Protein Production and Purification. The proteins were produced in E. coli TOP10 cells as $\mathrm{N}$-terminal histidine tagged recombinant proteins. The cells were grown in $2 \mathrm{~L}$ bottles with $500 \mathrm{~mL}$ of $2 \mathrm{xTY}$ at $30-37^{\circ} \mathrm{C}$ under vigorous shaking until the $\mathrm{OD}_{600 \mathrm{~nm}}$ reached $\sim 0.5$, after which the cells were induced with $0.02 \%(\mathrm{w} / \mathrm{v})$ L-arabinose and the growth was carried out at RT for 15-19 h. The cells were collected and suspended in A-buffer [ $50 \mathrm{mM}$ sodium phosphate, 100 $\mathrm{mM} \mathrm{NaCl}, 5 \mathrm{mM}$ imidazole, $10 \%$ (v/v) glycerol]. The cells were lysed by French press (SLM Aminco) or sonication, and the cell debris was removed by centrifugation. The crude lysate was mixed with TALON Superflow (GE Healthcare). After a wash step, the target proteins were eluted from the column with B-buffer (A-buffer with $250 \mathrm{mM}$ imidazole). The proteins were desalted with a PD-10 column (GE Healthcare) and stored at $-20{ }^{\circ} \mathrm{C}$ in C-buffer [ $100 \mathrm{mM}$ sodium phosphate, $100 \mathrm{mM} \mathrm{NaCl}$ ] with glycerol added to $40 \%(\mathrm{v} / \mathrm{v})$. The proteins SnogG and SnogA were supplemented with $1 \%$ of Triton X$100(\mathrm{v} / \mathrm{v})$ after the lysis of the cells or to C-buffer, respectively. The proteins were analyzed by SDS-PAGE (Figure S1) and photometrically at $280 \mathrm{~nm}$ (NanoDrop2000; Thermo Scientific).

Enzymatic Reactions. The reactions were performed in two steps. The first reaction was the synthesis of the dimethylated TDP-Lrhodosamine, while the second step was the attachment of the synthesized TDP-sugar to 14, obtained from earlier studies, ${ }^{16}$ via an $\mathrm{O}-\mathrm{C}$ and a $\mathrm{C}-\mathrm{C}$ bond.

The first reaction was performed with $30 \mu \mathrm{M}$ of RmlB, SnogI, SnogF, SnogG, SnogX, and SnogA and $10 \mu \mathrm{M}$ of SnogH immobilized in a
TALON Superflow (GE Healthcare) at $4{ }^{\circ} \mathrm{C}$ for $30 \mathrm{~min}$, or by omitting SnogA or SnogX. After immobilizing the enzymes, the master mix [buffer $\mathrm{pH} 7.5 ; 50 \mathrm{mM} \mathrm{Na} 3 \mathrm{PO}_{4} \cdot 12 \mathrm{H}_{2} \mathrm{O}, 100 \mathrm{mM} \mathrm{NaCl}, 5 \%$ (v/v) glycerol, $90 \mu \mathrm{M}$ pyridoxal 5'-phosphate (PLP; Sigma-Aldrich), $2 \mathrm{mM} \mathrm{S-}$ adenosylmethionine (SAM; Sigma-Aldrich), $6 \mathrm{mM}$ nicotinamide adenine dinucleotide (NAD+; Sigma-Aldrich), $3 \mathrm{mM}$ L-glutamate ,and $10 \mathrm{mM}$ thymidine-5'-diphospho- $\alpha$-D-glucose (TDP- $\alpha$-D-glucose; Carbosynth)] was added and incubated at $23{ }^{\circ} \mathrm{C}$ for $2 \mathrm{~h}$ with gentle shaking. The reaction mixture was centrifuged at $700 \mathrm{~g}$ for $10 \mathrm{~min}$ at 4 ${ }^{\circ} \mathrm{C}$, and the supernatant was carefully removed and analyzed by LC-MS (Agilent 1260 Infinity 6120 Quadropole LC/MS) using the Phenomenex synergy fusion RP $(4 \mu \mathrm{m}, 4.6 \times 150 \mathrm{~mm})$ column and a gradient from $0.1 \%$ formic acid to $100 \%$ acetonitrile.

The second reaction was achieved with a reaction containing buffer at $\mathrm{pH} 8,\left[50 \mathrm{mM} \mathrm{Na}_{3} \mathrm{PO}_{4} \cdot 12 \mathrm{H}_{2} \mathrm{O}, 100 \mathrm{mM} \mathrm{NaCl}, 5 \%\right.$ (v/v) glycerol], 7 $\mu \mathrm{M}$ SnogD, $2.5 \mu \mathrm{M}$ SnoK, $6 \mathrm{mM} 8$ (the concentration was estimated

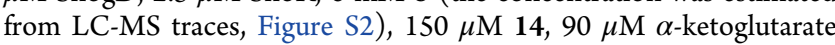
(aKG), $100 \mu \mathrm{M} \mathrm{Fe}(\mathrm{II}) \mathrm{SO}_{4}$, and $200 \mu \mathrm{M}$ L-ascorbate. The reaction was performed in $200 \mu \mathrm{L}$ for $3 \mathrm{~h}$ at $30^{\circ} \mathrm{C}$ with gentle shaking. The reaction was extracted with chloroform and analyzed by HPLC using a reverse phase column (Phenomenex Kinetics, $2.6 \mu \mathrm{m}, 4.6 \times 100 \mathrm{~mm}$ ) using a gradient from $15 \%$ acetonitrile with $0.1 \%$ formic acid to $100 \%$ acetonitrile. The reaction product was compared to authentic standards 5 and 15 obtained from previous studies. ${ }^{16,17}$ The yield of the reaction was $10.4 \%$ as calculated based on integration of HPLC peaks at $256 \mathrm{~nm}$ where the absorbance of the substrate and product are comparable.

\section{ASSOCIATED CONTENT}

\section{Supporting Information}

The Supporting Information is available free of charge on the ACS Publications website at DOI: 10.1021/acschembio. 8 b00658.

The SDS-PAGE gel of the enzymes used in this study (Figure S1); the purity of $\mathbf{8}$, shown by LC/MS (Figure S2); the list of the primers used in this study (Table S1) (PDF)

\section{AUTHOR INFORMATION}

\section{Corresponding Author}

*Tel.: +35823336847. Fax: +35823336860. E-mail: mianme@ utu.fi.

ORCID

Benjamin Nji Wandi: 0000-0003-1071-3111 Mikko Metsä-Ketelä: 0000-0003-3176-2908

Notes

The authors declare no competing financial interest.

\section{ACKNOWLEDGMENTS}

The financial support from the Academy of Finland (grant number 285971 to M.M-K.), the Alfred Kordelin Foundation, and the Finnish Cultural Foundation (the Central Foundation and Varsinais-Suomi Regional Funds; to V.S.) is acknowledged. We thank V. Trapp for assistance with protein production and purification.

\section{REFERENCES}

(1) Weymouth-Wilson, A. C. (1997) The role of carbohydrates in biologically active natural products. Nat. Prod. Rep. 14, 99-110.

(2) Arcamone, F., Cassinelli, G., Fantini, G., Grein, A., Orezzi, P., Pol, C., and Spalla, C. (1969) Adriamycin, 14-hydroxydaunomycin, a new antitumor antibiotic from S. peucetius var. caesius. Biotechnol. Bioeng. 11, $1101-1110$

(3) Schlünzen, F., Zarivach, R., Harms, J., Bashan, A., Tocilj, A., Albrecht, R., Yonath, A., and Franceschi, F. (2001) Structural basis for 
the interaction of antibiotics with the peptidyl transferase centre in eubacteria. Nature 413, 814-821.

(4) Mrozik, H., Linn, B. O., Eskola, P., Lusi, A., Matzuk, A., Preiser, F. A., Ostlind, D. A., Schaeffer, J. M., and Fisher, M. H. (1989) Syntheses and Biological Activities of 13-Substituted Avermectin Aglycons. J. Med. Chem. 32, 375-381.

(5) Kren, V., and Martínková, L. (2001) Glycosides in medicine: “The role of glycosidic residue in biological activity. Curr. Med. Chem. 8, $1303-1328$.

(6) Hultin, P. G. (2005) Bioactive C-glycosides from bacterial secondary metabolism. Curr. Top. Med. Chem. 5, 1299-1331.

(7) Bhuyan, B. K., and Dietz, A. (1965) Fermentation, taxonomic, and biological studies on nogalamycin. Antimicrob. Agents Chemother. 5, 836-844.

(8) Takahashi, H., Liu, Y. N., Chen, H., and Liu, H. W. (2005) Biosynthesis of TDP-L-mycarose: The specificity of a single enzyme governs the outcome of the pathway. J. Am. Chem. Soc. 127, 93409341.

(9) Melançon, C. E., 3rd, Hong, L., White, J. A., Liu, Y. N., and Liu, H. W. (2007) Characterization of TDP-4-keto-6-deoxy-D-glucose-3,4ketoisomerase from the D-mycaminose biosynthetic pathway of Streptomyces fradiae: In vitro activity and substrate specificity studies. Biochemistry 46, 577-590.

(10) Hong, L., Zhao, Z., Melançon, C. E., 3rd, Zhang, H., and Liu, H. W. (2008) In vitro characterization of the enzymes involved in TDP-Dforosamine biosynthesis in the spinosyn pathway of Saccharopolyspora spinosa. J. Am. Chem. Soc. 130, 4954-4967.

(11) Kharel, M. K., Lian, H., and Rohr, J. (2011) Characterization of the TDP-D-ravidosamine biosynthetic pathway: one-pot enzymatic synthesis of TDP-D-ravidosamine from thymidine-5-phosphate and glucose-1-phosphate. Org. Biomol. Chem. 9, 1799-1808.

(12) Wang, G., Kharel, M. K., Pahari, P., and Rohr, J. (2011) Investigating mithramycin deoxysugar biosynthesis: Enzymatic total synthesis of TDP-D-olivose. ChemBioChem 12, 2568-2571.

(13) Thibodeaux, C. J., Melançon, C. E., 3rd, and Liu, H. (2008) Natural-product sugar biosynthesis and enzymatic glycodiversification. Angew. Chem., Int. Ed. 47, 9814-9859.

(14) Singh, S., Phillips, G. N., Jr., and Thorson, J. S. (2012) The Structural Biology of Enzymes Involved in Natural Product Glycosylation. Nat. Prod. Rep. 29, 1201-1237.

(15) Bates, M. A., and Sammes, P. G. (1983) Total Synthesis of CGlycoside Fragment of Nogalamycin. J. Chem. Soc., Chem. Commun. O, 896-898.

(16) Siitonen, V., Claesson, M., Patrikainen, P., Aromaa, M., Mäntsälä, P., Schneider, G., and Metsä-Ketelä, M. (2012) Identification of LateStage Glycosylation Steps in the Biosynthetic Pathway of the Anthracycline Nogalamycin. ChemBioChem 13, 120-128.

(17) Siitonen, V., Selvaraj, B., Niiranen, L., Lindqvist, Y., Schneider, G., and Metsä-Ketelä, M. (2016) Divergent non-heme iron enzymes in the nogalamycin biosynthetic pathway. Proc. Natl. Acad. Sci. U. S. A. 113, 5251-5256.

(18) Metsä-Ketelä, M., Niemi, J., Mäntsälä, P., and Schneider, G. (2008) Anthracycline Biosynthesis: Genes, Enzymes and Mechanisms In Anthracycline Chemistry and Biology I: Biological Occurrence and Biosynthesis, Synthesis and Chemistry, (Krohn, K., Ed.) pp 101-140, Springer-Verlag, Berlin/Heidelberg.

(19) Grocholski, T., Dinis, P., Niiranen, L., Niemi, J., and MetsäKetelä, M. (2015) Divergent evolution of an atypical S-adenosyl-Lmethionine-dependent monooxygenase involved in anthracycline biosynthesis. Proc. Natl. Acad. Sci. U. S. A. 112, 9866-9871.

(20) Gui, C., Mo, X., Gu, Y. C., and Ju, J. (2017) Elucidating the Sugar Tailoring Steps in the Cytorhodin Biosynthetic Pathway. Org. Lett. 19, 5617-5620.

(21) Leimkuhler, C., Fridman, M., Lupoli, T., Walker, S., Walsh, C. T., and Kahne, D. (2007) Characterization of rhodosaminyl-transfer by the AknS/AknT glycosylation complex and its use in reconstituting the biosynthetic pathway of Aclacinomycin A. J. Am. Chem. Soc. 129, 10546-10550.
(22) Torkkell, S., Kunnari, T., Palmu, K., Mäntsälä, P., Hakala, J., and Ylihonko, K. (2001) The entire nogalamycin biosynthetic gene cluster of Streptomyces nogalater: characterization of a 20-kb DNA region and generation of hybrid structures. Mol. Genet. Genomics 266, 276-288.

(23) Stevenson, G., Neal, B., Liu, D. a N., Hobbs, M., Packer, N. H. Batley, M., Redmond, J. W., Lindquist, L., and Reeves, P. (1994) Structure of the 0 Antigen of Escherichia coli K-12 and the Sequence of Its $r I b$ Gene Cluster. J. Bacteriol. 176, 4144-4156.

(24) Sheldon, R. A., and Pereira, P. C. (2017) Biocatalysis engineering: the big picture. Chem. Soc. Rev. 46, 2678-2691.

(25) Claesson, M., Siitonen, V., Dobritzsch, D., Metsä-Ketelä, M., and Schneider, G. (2012) Crystal structure of the glycosyltransferase SnogD from the biosynthetic pathway of nogalamycin in Streptomyces nogalater. FEBS J. 279, 3251-3263.

(26) Moncrieffe, M. C., Fernandez, M.-J., Spiteller, D., Matsumura, H., Gay, N. J., Luisi, B. F., and Leadlay, P. F. (2012) Structure of the glycosyltransferase EryCIII in complex with its activating P450 homologue EryCII. J. Mol. Biol. 415, 92-101.

(27) Lu, W., Leimkuhler, C., Gatto, G. J., Kruger, R. G., Oberthür, M., Kahne, D., and Walsh, C. T. (2005) AknT is an activating protein for the glycosyltransferase AknS in L-aminodeoxysugar transfer to the aglycone of aclacinomycin A. Chem. Biol. 12, 527-534.

(28) Melançon, C. E., 3rd, Takahashi, H., and Liu, H. (2004) Characterization of tylM3/tylM2 and mydC/mycB pairs required for efficient glycosyltransfer in macrolide antibiotic biosynthesis. J. Am. Chem. Soc. 126, 16726-16727.

(29) Hong, J. S. J., Park, S. J., Parajuli, N., Park, S. R., Koh, H. S., Jung, W. S., Choi, C. Y., and Yoon, Y. J. (2007) Functional analysis of desVIII homologues involved in glycosylation of macrolide antibiotics by interspecies complementation. Gene 386, 123-130.

(30) Useglio, M., Peirú, S., Rodríguez, E., Labadie, G. R., Carney, J. R., and Gramajo, H. (2010) TDP-L-megosamine biosynthesis pathway elucidation and megalomicin a production in Escherichia coli. Appl. Environ. Microbiol. 76, 3869-3877.

(31) Nguyen, H. C., Karray, F., Lautru, S., Gagnat, J., Lebrihi, A., Ho Huynh, T. D., and Pernodet, J.-L. (2010) Glycosylation steps during spiramycin biosynthesis in Streptomyces ambofaciens: involvement of three glycosyltransferases and their interplay with two auxiliary proteins. Antimicrob. Agents Chemother. 54, 2830-2839.

(32) Kieser, T., Bibb, M. J., Buttner, M. J., Chater, K. F., and Hopwood, D. A. (2000) Practical Streptomyces Genetics, John Innes Foundation, Norwich, UK.

(33) Chater, K. F., and Wilde, L. C. (1980) Streptomyces albus G mutants defective in the SalGI restriction-modification system. Microbiology 116, 323-334.

(34) Kallio, P., Sultana, A., Niemi, J., Mäntsälä, P., and Schneider, G. (2006) Crystal structure of the polyketide cyclase AknH with bound substrate and product analogue: Implications for catalytic mechanism and product stereoselectivity. J. Mol. Biol. 357, 210-220.

(35) Datsenko, K. A., and Wanner, B. L. (2000) One-step inactivation of chromosomal genes in Escherichia coli K-12 using PCR products. Proc. Natl. Acad. Sci. U. S. A. 97, 6640-6645.

(36) Hoang, T. T., Karkhoff-Schweizer, R. R., Kutchma, A. J., and Schweizer, H. P. (1998) A broad-hostrange Flp-FRT recombination system for site-specific excision of chromosomally located DNA sequences: Application for isolation of unmarked Pseudomonas aeruginosa mutants. Gene 212, 77-86. 